\title{
Pre-Operative Anaemia Predicts Poor Outcomes in Patients Undergoing Infra-Inguinal Bypass Surgery
}

\author{
K. Sierakowski', Y. T. Wong1, C. Delaney ${ }^{1}$, P. Hakendorf ${ }^{2,3}$, K. Missen ${ }^{1}$, J. I. Spark1 \\ ${ }^{1}$ Department of Vascular Surgery, Flinders University, Bedford Park, South Australia, Australia \\ ${ }^{2}$ Clinical Epidemiology Unit, Flinders Medical Centre, Bedford Park, South Australia, Australia \\ ${ }^{3}$ Flinders Centre for Epidemiology and Biostatistics, Flinders University, Bedford Park, South Australia, Australia \\ Email: kyrasiera@gmail.com
}

Received 6 September 2014; revised 22 October 2014; accepted 5 November 2014

Copyright (C) 2014 by authors and Scientific Research Publishing Inc.

This work is licensed under the Creative Commons Attribution International License (CC BY). http://creativecommons.org/licenses/by/4.0/

(c) (1) Open Access

\section{Abstract}

Objectives: Critical limb ischaemia (CLI) requiring infra-inguinal bypass is a common presentation to the vascular surgery service. A significant number of CLI patients also present with anaemia as a comorbidity, the impact of which is unknown. We reviewed the impact of anaemia on mortality and amputation in patients who underwent infra-inguinal bypass surgery. Methods: Demographic data from all patients who underwent infra-inguinal bypass surgery for CLI at the Flinders Medical Centre \& Repatriation General Hospital, South Australia between January 2005 and January 2010 were recorded. Haemoglobin (Hb) level, serum creatinine (SCr) level, mortality and amputation events were also recorded for retrospective analysis. Results: 190 patients (141M, 49F) who underwent infra-inguinal bypass surgery over a 5 year period were analyzed. $100(53 \%)$ of these patients had mild anaemia (mean Hb $113 \mathrm{~g} / \mathrm{L}$, mean for females $103 \mathrm{~g} / \mathrm{L}$, mean for males $116 \mathrm{~g} / \mathrm{L}$ ). Those who presented with anaemia were more likely to have diabetes and renal dysfunction as comorbidities. Anaemia had a significant negative impact on mortality with $30 \%$ deaths in those who were anaemic compared to $15.6 \%$ deaths in those without anaemia odds ratio (OR) $1.76,95 \%$ CI: $0.90-3.48, p<0.01$. This relationship was similar when looking at amputation as an outcome; with the number of amputations in those who were anaemic preoperatively $14 \%$ vs $6.7 \%$ in those who were not anaemic, OR $2.56,95 \% \mathrm{CI}$ : $1.00-6.54, \mathrm{p}=0.05$. The negative impact of anaemia on mortality $43.5 \%$ vs $18.8 \%$ was also noted in patients who did not have diabetes or impaired renal function as a comorbidity $p<\mathbf{0 . 0 5}$. Conclusions: Anaemia is a common comorbidity in patients presenting with CLI and it predicts poor outcomes after infra-inguinal bypass. The role of transfusion is debatable, but it is clear that this group of patients requires careful management during the perioperative period to ensure they are medically optimized. 


\section{Keywords}

\section{Anaemia, Infra-Inguinal Bypass, Renal Impairment, Amputation, Mortality}

\section{Introduction}

Peripheral arterial disease (PAD) presenting as critical limb ischaemia (CLI) is increasingly common in an aging population. In a cross sectional study from Sweden, the prevalence of PAD and CLI increases with age, and up to $3.3 \%$ of the population aged 80 - 84 years old has CLI [1]. Many CLI patients are current or ex-smokers and suffer from multiple medical comorbidities including malignancies, diabetes, impaired renal function and poor nutrition, placing them at a high risk for developing anaemia of chronic disease [2]. Diabetes, renal impairment and smoking are recognized as independent risk factors for poor surgical outcomes [3]-[5]. Anaemia impairs the oxygen carrying capacity of the blood and may impair wound healing leading to increased risk of infective complications and may eventually lead to amputation. It may also impair other vital organ functions and the immune response resulting in increased mortality. Musallam et al. recently described mild anaemia as an independent predictor of increased thirty days morbidity and mortality in a large cohort of patients undergoing major non-cardiac surgery [2]. The unadjusted mortality rate in patients who had any degree of anaemia was six times that of those who did not have pre-operative anaemia [2].

The impact of pre-operative anaemia on patients presenting with critical limb ischaemia requiring lower extremity bypasses has not been previously studied. The aim of this retrospective case controlled study was to ascertain the impact of anaemia on the outcome of infra-inguinal bypasses in patients with CLI presenting to a tertiary referral hospital in South Australia.

\section{Methods}

Ethical approval was attained from the Southern Adelaide Clinical Human Research Ethics Committee (SAC HREC). All patients with Rutherford category 4, 5 \& 6 (CLI) who presented to the Southern Adelaide Health Services (Flinders Medical Centre, Bedford Park and Repatriation General Hospital, Daw Park, South Australia) between January 2005 and January 2010 and subsequently underwent infra-inguinal bypasses for treatment of CLI were identified from the Vascular Surgery Unit audit database (2005-2008) and the Australian Vascular Audit Database (2009-2010) and included in this retrospective study. Demographic data on age, gender, diabetes, hypertension, chronic heart failure (CHF), ischaemic heart disease (IHD), serum creatinine on admission (SCr) and smoking status (current or past) were recorded. Criteria for diagnosing anaemia were based on the Institute of Medical and Veterinary Sciences (IMVS) Pathology laboratory criteria. A haemoglobin level of less than 135 $\mathrm{g} / \mathrm{L}$ in males and less than $120 \mathrm{~g} / \mathrm{L}$ in females is diagnosed as anaemia. End points recorded were major amputation and all-cause mortality. Major amputation is defined as amputation proximal to metatarsal level. Our institution has a policy of preferentially using vein as a conduit for bypass surgery including the use of arm vein prior to resorting to prosthetic bypass with Miller's vein cuff. The exact techniques used during surgery were at the discretion of the consultant surgeon present at the case. Statistical analysis was performed with the assistance of a biostatistician using the STATA statistical software package. Chi-square tests were used to compare dichotomous end points, Kaplan-Meier survival curve was used to compare mortality data and log rank test was used to compare time to major amputation.

\section{Results}

There were 230 infra-inguinal bypasses performed for CLI between January 2005 and January 2010. Forty patients had no follow up information available post operatively and these patients were excluded from analysis. There was no difference in the demographic of those who had incomplete data set compared to those with complete data set. A total of 190 patients with complete data set were analyzed, demographic information is shown in Table 1. The mean follow-up period was 24.7 months. There was a preponderance of men in our patient population with distribution between the genders being 49 women (26\%) and 141 men (74\%). Of the patients who presented with CLI 100 (53\%) were anaemic. Patients who were anaemic were likely to be older than those who 
Table 1. Demographics of patients who underwent infra-inguinal bypasses 2005-2010.

\begin{tabular}{cccc}
\hline Demographic & Anaemic Group N = 100 (53\%) & Non-Anaemic Group N = 90 (47\%) & p Value \\
\hline Mean Age & 75 y.o & 71 y.o & 0.01 \\
Sex & $75 \mathrm{M} 25 \mathrm{~F}$ & $66 \mathrm{M} 24 \mathrm{~F}$ & 0.79 \\
Mean Pre-Op Hb & $113 \mathrm{~g} / \mathrm{L}$ & $144 \mathrm{~g} / \mathrm{L}$ & $<0.001$ \\
Renal Dysfunction $(\mathrm{SCr}>110 \mu \mathrm{mol} / \mathrm{L})$ & 40 & 10 & $<0.001$ \\
Renal Failure $(\mathrm{SCr}>300 \mu \mathrm{mol} / \mathrm{L})$ & 9 & 1 & 0.015 \\
Diabetes & 51 & 33 & 0.046 \\
\hline
\end{tabular}

were not anaemic with the average age of the anaemic group being four years older (75 y.o vs. 71 y.o; $p=0.01$ ). The patients who were classified as anaemic had a mean Hb level of $113 \mathrm{~g} / \mathrm{L} \pm \mathrm{SD} 14$. Females had an average of $103 \mathrm{~g} / \mathrm{L} \pm \mathrm{SD} 11$ and males $116 \mathrm{~g} / \mathrm{L} \pm \mathrm{SD} 14$.

Patients found to have anaemia on admission were more likely to have death (30\% vs $15.6 \%$, OR 1.76, 95\% CI: 0.90 - 3.48, p < 0.01) and amputation (14\% vs 6.7\%, OR 2.56, 95\% CI: $1.00-6.54$, p = 0.051) as an outcome compared to patients who were not anaemic (Figure 1 \& Figure 2). The median time between the patient's operation and their subsequent death was 558 days for the whole cohort, 314 days if they were anaemic, 1043 days if they were non-anaemic. The median time between a patient's operation and subsequent amputation was 78.5 days for all patients who underwent amputation, 78.5 days for those with anaemia and 121 days for those without anaemia.

Subgroup-analysis revealed that the impact of anaemia on patients who underwent infra-inguinal bypass is not limited to patients who had impaired renal function and/or diabetes $(n=105)$. In patients who did not have either diabetes or impaired renal function $(n=85)$, the presence of anaemia preoperatively increased mortality (36.1\%, vs $12.2 \%, \mathrm{P}<0.01)$ but not amputation rate $(8.3 \%$ vs $12.2 \%, \mathrm{p}=0.58)$ compared with those who were not anaemic (Table 2 \& Table 3). The presence of diabetes alone did not increase mortality (22.4\% vs $23.6 \%$, p $\geq$ $0.8)$ or amputation rate $(11.6 \%$ vs $9 \%, \mathrm{p}=0.63)$. Similarly the presence of impaired renal function (SCr $>120$ $\mu \mathrm{mol} / \mathrm{L})$ alone did not increase mortality (25.7\% vs $23 \%, \mathrm{p}=0.36)$ or amputation $(11.4 \%$ vs $10.5 \%, \mathrm{p}=0.43)$.

Subgroup analysis was performed for age but not for gender, as there was no significant difference between genders was demonstrated between the two cohorts. The group who suffered from anaemia was on average four years older, however there was no significant difference in the mortality rate of patients who had anaemia aged 70 years and older $(n=73)$ compared to those aged less than 70 years old $(n=27)(34 \%$ vs $30 \%, p=0.66)$.

\section{Discussion}

Our study has demonstrated that anaemia is a common comorbidity in patients presenting with CLI. Fifty three percent of our patients with CLI had anaemia on admission. This is in line with a study published by Shah M. et al. who found that $56 \%$ of patients admitted to a tertiary hospital in the United Kingdom for CLI had anaemia [2]. Most of our patients with anaemia as a comorbidity on admission had only mild anaemia (mean 113 g/L \pm SD 14).

Our study also showed that patients who presented with anaemia were more likely to have diabetes and renal failure as comorbidities and therefore it could be reasonably argued that the poor outcome is a reflection of their comorbidities. Our findings suggested that anaemia in the absence of diabetes or renal dysfunction also increased mortality rate (36.1\% vs 12.2\%) during a mean follow up period of 24.7 months (range 1 day - 75.6 months). These findings are supported by the study by Elmistekawy et al. who found that preoperative anaemia was an independent risk factor for post operative morbidity and mortality in patients undergoing aortic valve replacements [6].

Diabetes or renal failure alone in this retrospective study has not been associated with a worse outcome in mortality or amputation rates. This is not consistent with previous studies that have demonstrated worse outcomes in vascular surgery in the presence of renal failure as a comorbidity [3] [7]. The definition for renal dysfunction in our study was serum creatinine $>120 \mu \mathrm{mol} / \mathrm{L}$ therefore a significant proportion (63\%) of our patient cohort had only mild renal impairment (serum creatinine 120 - $180 \mu \mathrm{mol} / \mathrm{L}$ ). This mild level of renal impairment may not adversely affect surgical outcomes. It is likely that more severe renal impairment will negatively impact 


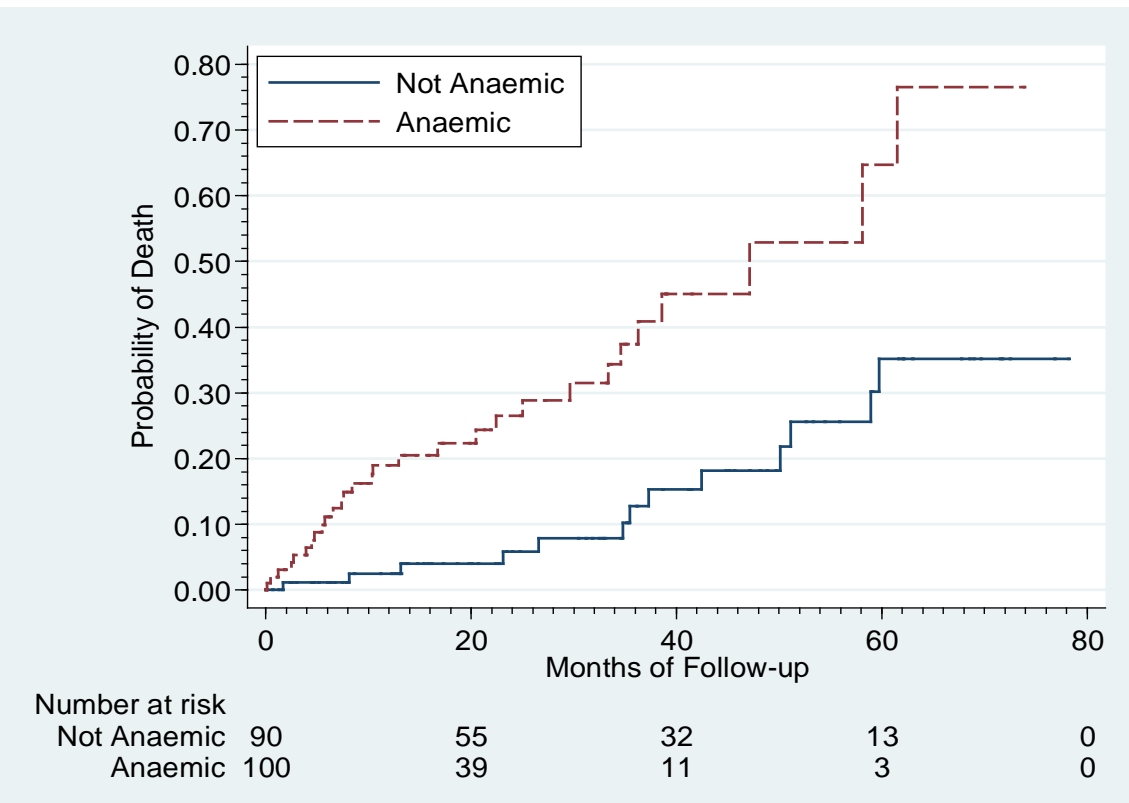

Figure 1. Mortality for patients with anaemia versus no anaemia.

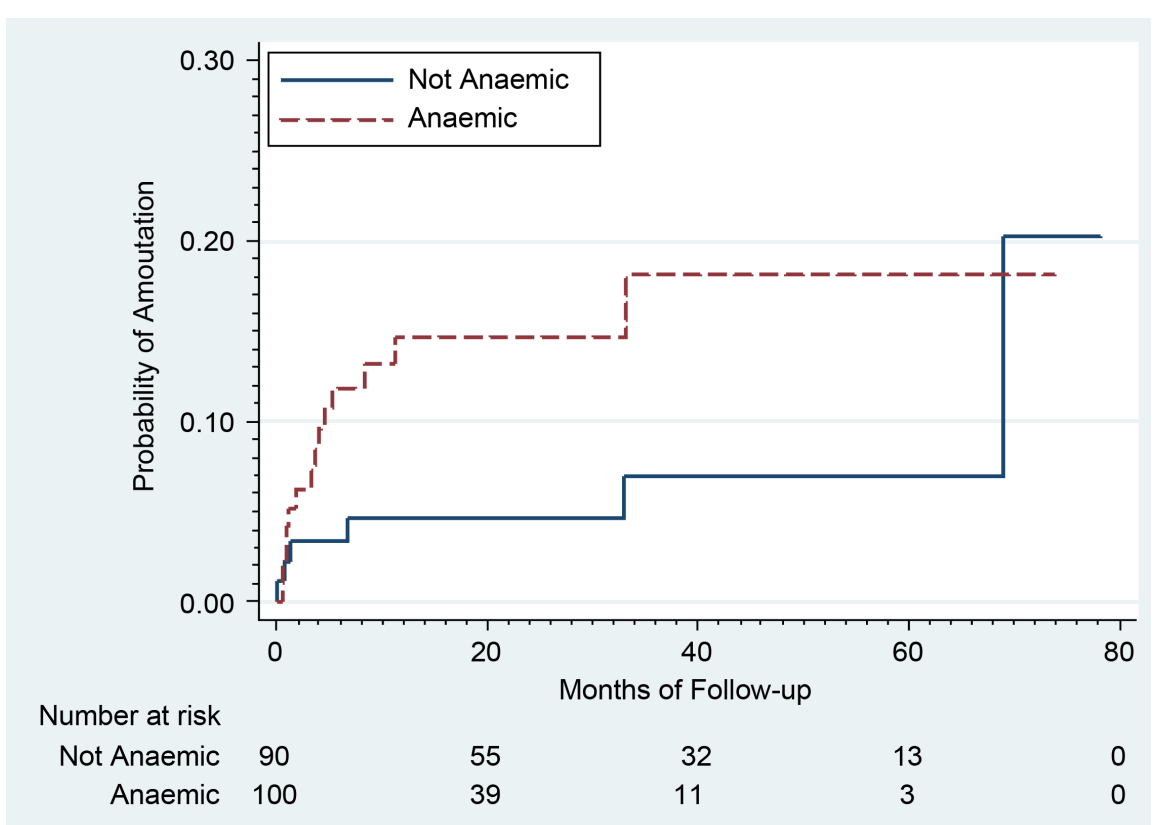

Figure 2. Amputation rates for patients with anaemia versus no anaemia.

Table 2. Risk of mortality following infra-inguinal bypass surgery in patients with anaemia but no diabetes or renal dysfunction.

\begin{tabular}{|c|c|c|c|c|}
\hline & & \multicolumn{2}{|c|}{ Anaemia } & \multirow{2}{*}{ Total } \\
\hline & & Non-Anaemic & Anaemic & \\
\hline \multirow{2}{*}{ Survival } & Alive & 43 & 26 & 76 \\
\hline & Dead & $6(12.2 \%)$ & 13 (36.1\%) & $19 \mathrm{P}=0.01$ \\
\hline \multicolumn{2}{|c|}{ Total } & 49 & 36 & 85 \\
\hline
\end{tabular}


Table 3. Risk of amputation following infra-inguinal bypass surgery in patients with anaemia but no diabetes or renal dysfunction.

\begin{tabular}{cccc}
\hline & \multicolumn{2}{c}{ Anaemia } & Total \\
\cline { 2 - 3 } & Non-Anaemic & Anaemic & 76 \\
No Amputation & 43 & 33 & $9, \mathrm{p}=0.58$ \\
Amputation & $6(12.2 \%)$ & $3(8.3 \%)$ & 85 \\
Total & 49 & 36 & 85 \\
\hline
\end{tabular}

outcomes. Diabetes in isolation ( $\mathrm{n}=62)$ does not necessarily impact on outcome [8] [9] and therefore it is not surprising that diabetes in isolation did not alter our results. Patients with CLI who have both diabetes and renal failure were more likely to have had diabetes for a prolonged period of time with multiple end organ damage, and thus anaemia did not add further risk to the all-cause mortality in our patient population.

Age could arguably be a contributory factor to the poorer outcome in patients with anaemia, however our study did not show a significant difference in the mortality rate in patients with anaemia presenting for bypass surgery due to CLI regardless of age ( $34 \%$ vs $30 \%, \mathrm{p}=0.66)$.

The pathophysiology of anaemia in patients with CLI is likely to be anaemia of chronic disease. The pathophysiology of anaemia of chronic disease is poorly understood and it is likely to be a combination of pathologic iron haemostasis, impaired erythropoiesis and blunted erythropoietin response [10]. Peripheral arterial disease is a chronic inflammatory state, and this could result in increased sequestration of iron in the reticuloendothelial system and decreased absorption of iron from the duodenum resulting in reduced availability of iron available for erythropoiesis [10]. Various cytokines produced in chronic inflammatory states can also inhibit production of red blood cells through direct cytotoxic effect on bone marrow, induction of apoptosis of erythroid progenitor cells and decreased erythropoietin expression by the kidney [10]. Treatment of anaemia in patients with CLI can be approached from several angles such as transfusion of red blood cells, direct iron supplementation to increase availability of iron for erythropoiesis, inhibition of inflammatory cytokines and erythropoietin supplementation.

Transfusion for anaemia has been extensively studied in the critical care setting [11]. Maintaining a Hb level greater than $100 \mathrm{~g} / \mathrm{L}$ has not been shown to have superior outcomes compared to a transfusion threshold of 70 g/L except perhaps for those with ischaemic heart disease. Liberal transfusion has been associated with multiorgan failure and increased mortality in patients who are in the critical care environment. Most of our patients only had mild anaemia and it is therefore unlikely that transfusion would change the outcomes in our patient population.

Iron therapy either in the form of oral iron or intravenous iron can be used to increase iron availability for erythropoiesis. Oral iron however is likely to be poorly absorbed in chronic inflammatory state such as severe peripheral arterial disease due to down regulation of absorption in the duodenum. Intravenous iron can also be used to increase iron availability, however this is not always readily available, it is more cumbersome to administer and there is potential for serious allergic reaction. Iron supplementation therapy may be effective and easily administered however there is a bigger concern with iron therapy in patients with CLI. Iron is an essential nutrient for proliferation of microorganisms and tumour cells. Sequestration of iron by the reticuloendothelial system is a natural defense mechanism against infection and neoplasia [12] [13].

The use of erythropoietin in reversing anaemia of chronic disease has been very successful in chronic kidney disease and to a lesser extent in patients with cancer undergoing chemotherapy. The concern with the liberal use of erythropoietin in patients with critical limb ischaemia is that erythropoietin receptors have been found in several malignant cell lines including breast, ovarian, uterine, prostate, liver, kidney and myeloid cell [10]. The expression of erythropoietin receptors by cancer cells has been linked to neo-angiogenesis and tumor infiltration [14]. The prevalence of malignancy in patients with critical limb ischaemia was $11.5 \%$ in a tertiary referral vascular surgery unit in the United Kingdom in one study [15]. The use of erythropoietin in patients with critical limb ischaemia raises concern for aggravating occult malignancy. Erythropoietin may be useful in patients without malignancy, however the time required to thoroughly investigate these patients to exclude malignancy and the effect of erythropoietin on haemoglobin level is only achieved after one to two weeks of administration [16] and most patients with critical limb ischaemia are likely to need urgent surgery, thus limiting the usefulness of erythropoietin. 


\section{Conclusion}

The presence of anaemia in patients with critical limb ischaemia presenting for surgery predicts poor outcome. Mild anaemia is likely a harbinger of severe chronic disease and this is unlikely to be reversed by transfusion or iron therapy. The usefulness of erythropoietin is limited by the time required for it to have an effect on anaemia as most patients needing infra-inguinal bypasses for critical limb ischaemia require urgent surgery. Its use is also of concern in those with increased risk of malignancy. Careful attention is required to thoroughly investigate these patients and treat their underlying illness and a multidisciplinary and holistic approach should be adopted to optimize their care, so that the best possible outcomes can be achieved.

\section{Acknowledgements}

The authors do not have any conflict of interest to disclose.

\section{References}

[1] Sigvant, B., Wiberg-Hedman, K., Bergqvist, D., Rolandsson, O., Andersson, B., Persson, E. and Wahlberg, E. (2007) A Population-Based Study of Peripheral Arterial Disease Prevalence with Special Focus on Critical Limb Ischemia and Sex Differences. Journal of Vascular Surgery, 45, 1185-1191. http://dx.doi.org/10.1016/j.jvs.2007.02.004

[2] Shah, M., Martin, A., Myers, B., MacSweeney, S. and Richards, T. (2010) Recognising Anaemia and Malnutrition in Vascular Patients with Critical Limb Ischaemia. Annals of the Royal College of Surgeons of England, 92, 495-498. http://dx.doi.org/10.1308/003588410X12664192075738

[3] Bakken, A.M., Palchik, E., Hart, J.P., Rhodes, J.M., Saad, W.E. and Davies, M.G. (2007) Impact of Diabetes Mellitus on Outcomes of Superficial Femoral Artery Endoluminal Interventions. Journal of Vascular Surgery, 46, 946-958.

[4] Tennyson, C., Lee, R. and Attia, R. (2013) Is There a Role for HbA1c in Predicting Mortality and Morbidity Outcomes after Coronary Artery Bypass Graft Surgery? Interactive Cardiovascular and Thoracic Surgery, 17, 1000-1008. http://dx.doi.org/10.1093/icvts/ivt351

[5] Owens, C.D., Ho, K.J., Kim, S., Schanzer, A., Lin, J., Matros, E., Belkin, M. and Conte, M.S. (2007) Refinement of Survival Prediction in Patients Undergoing Lower Extremity Bypass Surgery: Stratification by Chronic Kidney Disease Classification. Journal of Vascular Surgery, 45, 944-952. http://dx.doi.org/10.1016/j.jvs.2007.01.025

[6] Elmistekawy, E., Rubens, F., Hudson, C., McDonald, B., Ruel, M., Lam, K., Mesana, T. and Boodhwani, M. (2014) Preoperative Anaemia Is a Risk Factor for Mortality and Morbidity Following Aortic Valve Surgery. European Journal of Cardio-Thoracic Surgery, 44, 1051-1056.

[7] Owens, C.D., Ho, K.J., Kim, S., Schanzer, A., Lin, J., Matros, E., Belkin, M. and Conte, M.S. (2007) Refinement of Survival Prediction in Patients Undergoing Lower Extremity Bypass Surgery: Stratification by Chronic Kidney Disease Classification. Journal of Vascular Surgery, 45, 944-952. http://dx.doi.org/10.1016/j.jvs.2007.01.025

[8] Ballotta, E., Toniato, A., Piatto, G., Mazzalai, F. and Da Giau, G. (2014) Lower Extremity Arterial Reconstruction for Critical Limb Ischemia in Diabetes. Journal of Vascular Surgery, 59, 708-719. http://dx.doi.org/10.1016/j.jvs.2013.08.103

[9] Hertzer, N.R., Bena, J.F. and Karafa, M.T. (2007) A Personal Experience with the Influence of Diabetes and Other Factors on the Outcome of Infrainguinal Bypass Grafts for Occlusive Disease. Journal of Vascular Surgery, 46, 271279.

[10] Weiss, G. and Goodnough, L. (2005) Anemia of Chronic Disease. The New England Journal of Medicine, 352, 10111023. http://dx.doi.org/10.1056/NEJMra041809

[11] Herbert, P., Wells, G., Blajchman, M., Marshall, J., Martin, C., Pagliarello, G., Tweeddale, M., Schweitzer, I. and Yetisir, E. (2000) A Multicenter, Randomized, Controlled Clinical Trial of Transfusion Requirements in Critical Care. The New England Journal of Medicine, 340, 1-9.

[12] Gombotz, H. (2001) Patient Blood Management Is Key before Elective Surgery. The Lancet, 378, 1362-1363. http://dx.doi.org/10.1016/S0140-6736(11)61552-3

[13] Weinberg, E.D. (2010) The Hazards of Iron Loading. Metallomics, 2, 732. http://dx.doi.org/10.1039/c0mt00023j

[14] Acs, G., Acs, P. and Beckwith, S. (2001) Erythropoietin and Erythropoietin Receptor Expression in Human Cancer. Cancer Research, 61, 3561-3565.

[15] Sakka, E.K., Gambhir, R.P.S., Halawa, M., Chong, P. and Rashid, H. (2005) Association of Malignant Disease with Critical Leg Ischaemia. British Journal of Surgery, 92, 1498-1501. http://dx.doi.org/10.1002/bjs.5125

[16] AMGEN (2005) Arasnep Prescribing Information, 1-45. 


\section{Abbreviations}

$\mathrm{PAD}$, peripheral arterial disease;

SCr, serum creatinine level;

IHD, ischaemic heart disease;

$\mathrm{CHF}$, chronic heart failure;

M, male;

F, female;

$\mathrm{Hb}$, haemoglobin level;

CLI, critical limb ischaemia;

ALI, acute limb ischaemia;

GSV, great saphenous vein;

IMVS, Institute of Medical and Veterinary Sciences;

y.o, years old. 
Scientific Research Publishing (SCIRP) is one of the largest Open Access journal publishers. It is currently publishing more than 200 open access, online, peer-reviewed journals covering a wide range of academic disciplines. SCIRP serves the worldwide academic communities and contributes to the progress and application of science with its publication.

Other selected journals from SCIRP are listed as below. Submit your manuscript to us via either submit@scirp.org or Online Submission Portal.
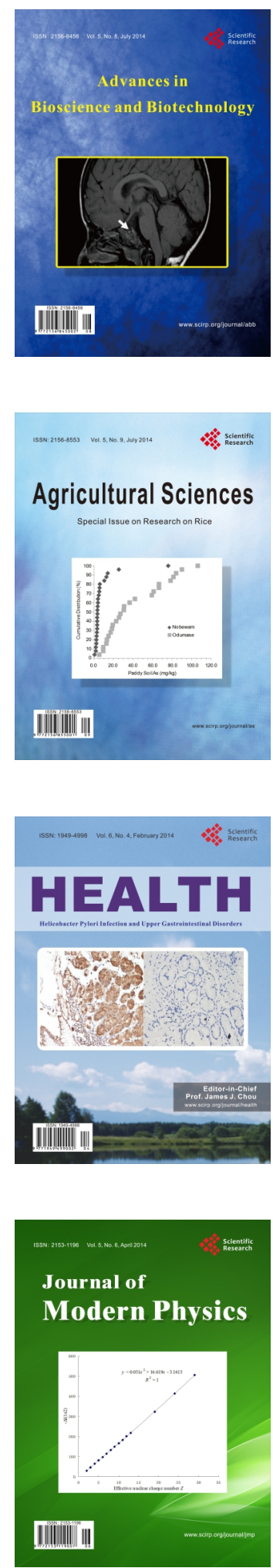
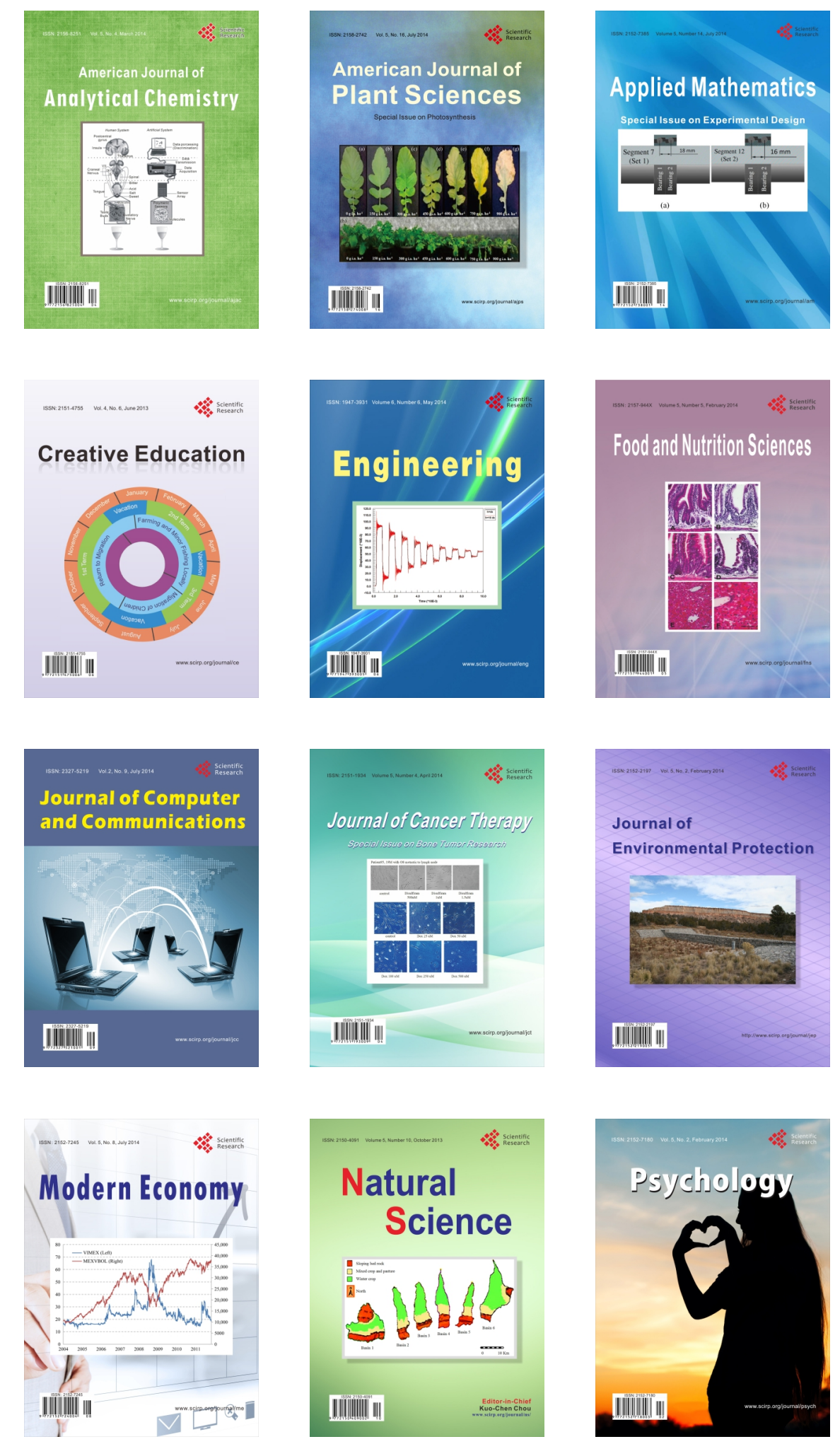